\title{
BMJ open Pharmacist-led management of chronic pain in primary care: results from a randomised controlled exploratory trial
}

\author{
Hanne Bruhn, ${ }^{1}$ Christine M Bond, ${ }^{2}$ Alison M Elliott, ${ }^{2}$ Philip C Hannaford, ${ }^{2}$ \\ Amanda J Lee, ${ }^{3}$ Paul McNamee, ${ }^{4}$ Blair H Smith, ${ }^{5}$ Margaret C Watson, ${ }^{2}$ \\ Richard Holland, ${ }^{6}$ David Wright ${ }^{7}$
}

To cite: Bruhn H, Bond CM, Elliott AM, et al. Pharmacistled management of chronic pain in primary care: results from a randomised controlled exploratory trial. BMJ Open 2013;3:e002361.

doi:10.1136/bmjopen-2012002361

- Prepublication history and additional material for this paper are available online. To view these files please visit the journal online (http://dx.doi.org/10.1136/ bmjopen-2012-002361)

Received 16 November 2012 Revised 6 February 2013 Accepted 7 February 2013

This final article is available for use under the terms of the Creative Commons Attribution Non-Commercial 2.0 Licence; see http://bmjopen.bmj.com

For numbered affiliations see end of article.

Correspondence to Professor C M Bond; c.m.bond@abdn.ac.uk

\section{ABSTRACT}

Objectives: To compare the effectiveness of pharmacist medication review, with or without pharmacist prescribing, with standard care, for patients with chronic pain.

Design: An exploratory randomised controlled trial.

Setting: Six general practices with prescribing pharmacists in Grampian (3) and East Anglia (3).

Participants: Patients on repeat prescribed pain medication (4815) were screened by general practitioners (GPS), and mailed invitations (1397). 196 were randomised and $180(92 \%)$ completed. Exclusion criteria included: severe mental illness, terminally ill, cancer related pain, history of addiction.

Randomisation and intervention: Patients were randomised using a remote telephone service to: (1) pharmacist medication review with face-to-face pharmacist prescribing; (2) pharmacist medication review with feedback to GP and no planned patient contact or (3) treatment as usual (TAU). Blinding was not possible.

Outcome measures: Outcomes were the SF-12v2, the Chronic Pain Grade (CPG), the Health Utilities Index 3 and the Hospital Anxiety and Depression Scale (HADS). Outcomes were collected at 0, 3 and 6 months.

Results: In the prescribing arm $(n=70)$ two patients were excluded/nine withdrew. In the review arm $(n=63)$ one was excluded/three withdrew. In the TAU arm $(n=63)$ four withdrew. Compared with baseline, patients had an improved CPG in the prescribing arm, $47.7 \%(21 / 44 ; p=0.003)$ and in the review arm, 38.6\% (17/44; $p=0.001)$, but not the TAU group, 31.3\% (15/ 48; ns). The SF-12 Physical Component Score showed no effect in the prescribing or review arms but improvement in TAU ( $p=0.02)$. The SF-12 Mental Component Score showed no effect for the prescribing or review arms and deterioration in the TAU arm $(p=0.002)$. HADS scores improved within the prescribing arm for depression $(p=0.022)$ and anxiety $(p=0.007)$, between groups $(p=0.022$ and $p=0.045$, respectively).

Conclusions: This is the first randomised controlled trial of pharmacist prescribing in the UK, and suggests that there may be a benefit for patients with chronic pain. A larger trial is required.

\section{ARTICLE SUMMARY}

Article focus

- Chronic pain (lasting $>3$ months) affects up to half the adult population, most of whom are primarily managed in primary care but prescribing is often suboptimal.

- Pharmacists now have prescribing rights but no published research has compared the effectiveness of their prescribing with that of GPs.

- The hypothesis was that pharmacist advice (with or without pharmacist prescribing) would lead to better outcomes than usual care.

Key messages

- The findings suggest that there may be improved pain-related outcomes for patients receiving pain-related care from a pharmacist prescriber.

- A larger trial is called for.

Strengths and limitations of this study

- This the first randomised controlled trial of pharmacist prescribing in the UK looking at patient reported clinical outcomes.

- The study was designed as an exploratory trial so no power calculation was done.

Trial registration: www.isrctn.org/ISRCTN06131530. Medical Research Council funding.

\section{INTRODUCTION}

Chronic pain (pain lasting more than 3 months) affects up to half the adult UK population, and is considered severely limiting in about $15 \%$ of cases. ${ }^{1}$ Recovery is uncommon with nearly $80 \%$ of those identified with chronic pain at baseline still reporting chronic pain 4 years later. ${ }^{2}$ It adversely affects many aspects of a person's physical and psychological health, and social and economic well-being. ${ }^{3-6}$

In the UK, most patients with chronic pain present, and are managed, in primary care. ${ }^{7}$ 
Although non-pharmacological treatments are available, these are accessed by few patients, with mixed success. ${ }^{8-10}$ Analgesics prescribed in primary care remain the mainstay of treatment, ${ }^{4}$ representing substantial workload and cost. Suboptimal prescribing may lead to poor pain control and other adverse patient outcomes. One study found that the most common medications involved in adverse drug reaction-related emergency admissions involved non-steroidal anti-inflammatory drugs (NSAIDs) ${ }^{11}$ which are commonly used to manage pain. Improved prescribing could result in better outcomes and remove the need for more costly, scarce, alternatives.

Pharmacists working in UK general practices are wellplaced to improve pain pharmacotherapy because of their expertise in therapeutics, understanding of the polypharmacy regimens ${ }^{12}$ frequently used in chronic pain management and established relationships with other primary care colleagues. In the UK National Health Service (NHS), recent regulatory changes now allow accredited pharmacists (as well as some other healthcare professionals such as nurses) to prescribe prescription-only medicines. ${ }^{13}$ Pharmacists can either be qualified as supplementary prescribers, in which case they operate within an agreed clinical management plan (CMP) in partnership with the doctor and patient, or as an independent prescriber, in which case they can either prescribe completely independently or within a CMP.

However, despite the increasing number of nonmedical prescribers, including pharmacists, there has been no rigourous comparisons of the outcomes of nonmedical versus general practitioner (GP) prescribing. This information is needed to assess the clinical effectiveness of different care models.

This paper reports findings from an exploratory randomised controlled trial (RCT) comparing pharmacist medication review, with or without pharmacist prescribing, with standard care for patients with chronic pain. Development of the trial was informed by earlier feasibility work. ${ }^{14} 15$

The a priori hypothesis was that, in patients with chronic pain, pharmacist advice (with or without pharmacist prescribing) would lead to better patient functioning and/or better pain control at 6 months, than treatment as usual (TAU).

\section{METHODS}

\section{Regulatory issues}

Ethical approval was granted by the National Research Ethics Service Committee-North of Scotland (reference number 09/S0801/107). NHS Research and Development approval was granted by NHS Grampian and East Norfolk \& Waveney Research Governance Committees. Patients gave informed consent before taking part.
Design

An open, exploratory RCT in which patients were randomised to one of the three study arms. Participants were not blind to allocated treatment arm due to the nature of the intervention.

\section{Recruitment of practices and independent prescribing pharmacists}

Practices in the Grampian Health Board area, Scotland $(n=18)$ and East Anglia region of England $(n=4)$ known to have an attached Royal Pharmaceutical Society of Great Britain registered independent pharmacist prescriber, were eligible to take part. From those indicating a willingness to participate, convenience sampling was used to identify six general practices: three in Grampian and three in East Anglia.

\section{Patient inclusion and exclusion criteria}

Patients registered with the recruited practices were eligible for inclusion if they were over 18 years of age, living in their own houses and receiving regular prescribed medication for pain. Patients were identified by a computerised search ${ }^{14}$ of the drug records of all individuals registered with the practice, to identify those who had received either two or more acute prescriptions, and/or one repeat prescription within the last 120 days, for an analgesic (British National Formulary (BNF section 4.7) and/or NSAID (BNF section 10.1.1). Medications that can be used for analgesia but whose primary indication is not chronic pain (eg triptans, antiepileptics or antidepressants) were excluded as these drugs identify few additional eligible patients. ${ }^{16}$ In accordance with trial criteria, GPs excluded and recorded reasons for patients who had: a concomitant severe mental health problem or terminal illness; had suffered recent bereavement; had a known alcohol or drug addiction; suffered pain caused by cancer or other malignancy; were unable to give informed consent; other (unspecified) reasons.

\section{Patient recruitment}

Eligible patients were sent an invitation pack (letter, information sheet, consent form) by practice staff between March and June 2010. Consent forms were returned directly to the researchers, who sent out a baseline questionnaire. Patients returning completed questionnaires were randomised by the researcher using a telephone randomisation service with a random number allocation which ensured allocation concealment. The allocation sequence was $1: 1: 1$.

\section{Intervention}

All participating pharmacists took part in a 2-day course updating them about pain management. As part of the training, participants defined and agreed the treatment algorithm they would all use. 
'Prescribing' arm

Pharmacists invited patients to a face-to-face consultation. Prior to the consultation, pharmacists completed a paper-based medication review of each patient's medical record and patients were asked to complete a pain diary to inform the consultation. A pharmaceutical care plan was agreed between the pharmacist and the patient. The plan assessed and documented relevant medical history and current conditions; known allergies and adverse drug reactions; relevant laboratory results; pain-related medications prescribed in the previous 10 years; current pain-related prescription medications; current symptoms; lifestyle issues, including units of alcohol consumed per week; recommendations for changes to medication (if any); whether nonpharmaceutical treatments had been considered; and any other relevant issues. Copies of the pain diary and pharmaceutical care plan are available from the authors on request. At the end of the consultation any required prescriptions for medicines were issued by the pharmacist. Owing to Controlled Drug (CD) regulations in place at the time, prescribing for CDs was performed using a supplementary prescribing $\mathrm{CMP},{ }^{17}$ rather than independent prescribing. Patients were followed up either by phone or face-to-face, at each pharmacist's discretion.

'Review arm': The pharmacists conducted a paperbased medication review focussed on pain-related prescription medications, before creating a pharmaceutical care plan which detailed any recommendations for medication changes. The plan was passed to the patient's GP for implementation. The GPs were asked subsequently about actions taken as a result of the recommendations.

Treatment as usual: Patients received standard general practice care.

\section{Outcome measures}

A core aim of this exploratory RCT was to finalise the selection of outcome measures for a subsequent multicentred RCT. In the Current Controlled Trials Registration (ISRCTNO6131530) we specified both primary and secondary outcome measures (primary: SF12, Health Utilities Index (HUI); secondary: chronic pain grade (CPG), HADS) based on our judgement following the earlier feasibility study. ${ }^{15}$ However in practice, all outcomes were considered equal and no single measure was defined as the primary outcome, for example, for the purpose of a sample size calculation (see below). These four outcome measures are described below. Inclusion of both pain-specific and generic outcome measures was based on Initiative on Methods, Measurement and Pain Assessment in Clinical Trials (IMMPACT) recommendations ${ }^{18}$ and an earlier feasibility study. ${ }^{15}$

The SF-12v2 is a generic health and functioning scale, ${ }^{19}$ previously used in population-based studies of pain. ${ }^{20} 21$ A Physical (PCS) and Mental Component
Score (MCS) were calculated, ranging from 0 to 100 ; a higher score indicates better functioning.

The HUI3 is a preference-based system for measuring comprehensive health status and health-related quality of life (HRQL). ${ }^{22}$ It provides descriptive evidence and a score for each dimension of health (vision, hearing, speech, ambulation/mobility, pain, dexterity, self-care, emotion and cognition) and an HRQL score for overall health. Each dimension has 3-6 levels. Owing to the cost of the additional license fee to score data from this measure, this instrument was not subsequently analysed.

The $\mathrm{CPG}^{23}$ is a seven-item scale which assesses pain severity on two dimensions: disability and intensity. The scale classifies pain according to the level of intensity and disability (I (low disability-low intensity) to IV (high disability-severely limiting)).

The Hospital Anxiety and Depression Scale (HADS) ${ }^{24}$ is a 14-item screening instrument which identifies the possible and probable caseness of anxiety (7 items (HADS-A)) and depression (7 items (HADS-D)); each item scored from 0 (not present) to 3 (highly present). Standard thresholds and previously used labels ${ }^{25}$ were applied: no depression/anxiety (0-7), mild (8-10), moderate $(11-15)$ or severe $(>15))$.

\section{Data collection}

\section{Participant questionnaires}

Questionnaires were posted to participants at baseline (pre-randomisation) and 3 and 6 months postrandomisation (follow-up was conducted between July 2010 and January 2011). Up to two reminders were sent. Questionnaire content included the outcome measures described earlier together with items on: demographic status (baseline only); screening items to confirm eligibility (baseline only); duration of pain condition (baseline only); location of pain; Morisky Medication Adherence Scale 4 (MMAS- 4$) ;{ }^{26}$ participant satisfaction (11 statements derived from the feasibility study for the prescribing arm (3 months only)) and additional comments by participants. The MMAS-4 provides a score of self-reported adherence to medication regimen. Scores range from 0 (low adherence) to 4 (high adherence).

\section{Follow-up interviews with staff}

Post-intervention, all pharmacists and all GPs in participating practices were invited to take part in semistructured interviews, carried out face-to-face when possible, otherwise by telephone. Interviews were taped, transcribed verbatim and content analysis was carried out.

\section{Sample size}

As this was an exploratory trial to estimate the effect size for a larger trial, no formal sample size calculation was possible. ${ }^{27}$ We aimed to recruit 30 participants per practice $(n=180$; with an additional six per practice for training purposes, that is, 216 in total). This was deemed sufficient to give reliable effect size estimates for the outcome measures of health status or CPG. 


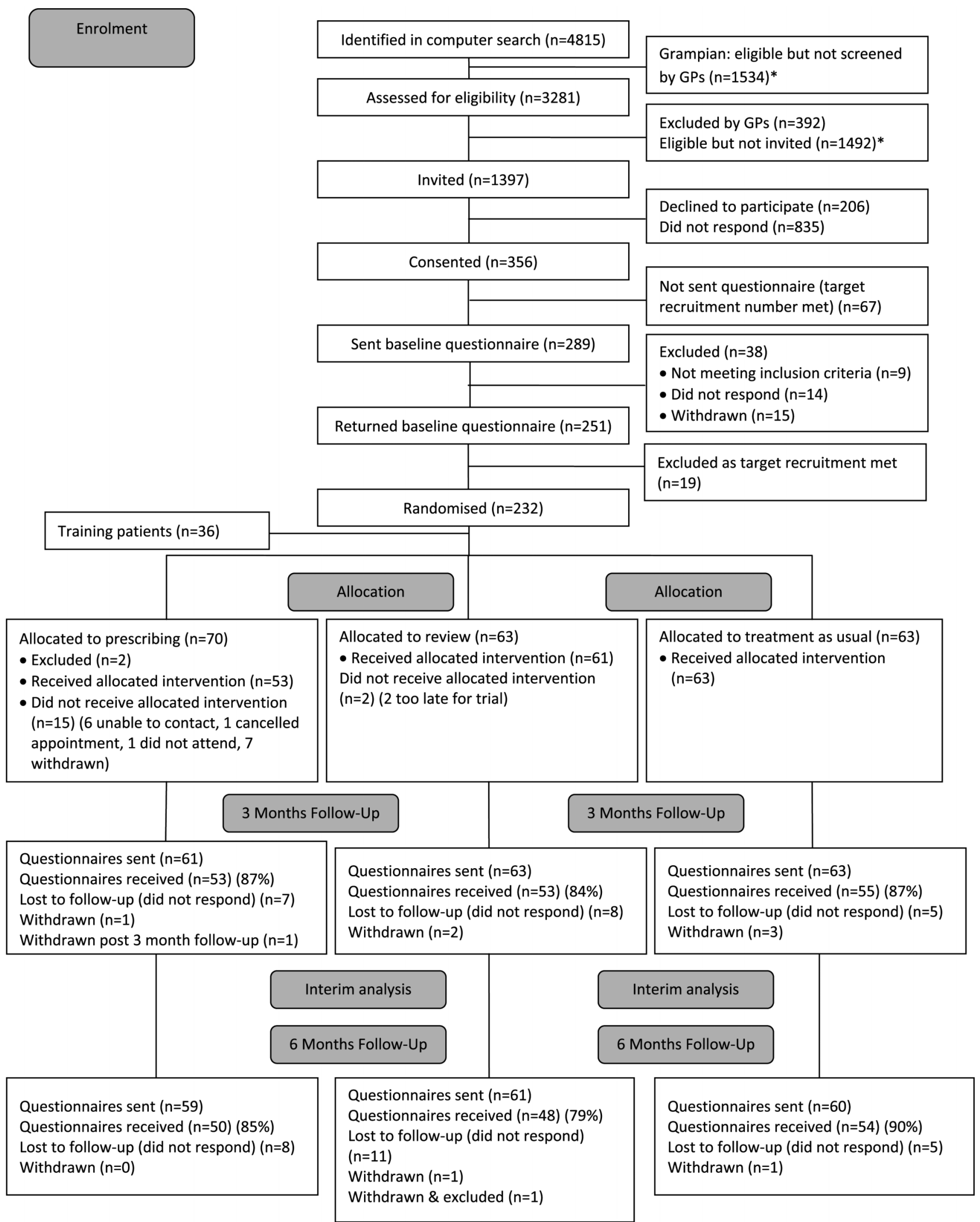

*In the Grampian Health Board area, on the basis of response rates in the earlier feasibility study (241 screened patients resulted in 22 recruited) only a random sample of eligible participants were screened (15). In East Anglia all eligible patients were screened.

Figure 1 CONSORT flow diagram. 
Data management and analysis

Data were entered into identical SPSS databases at each site and accuracy checks carried out on $10 \%$ before databases were merged. Descriptive statistics included means and SD for normally distributed continuous data, medians (IQR) for skewed continuous data and percentages $(n)$ for categorical data. Analysis was conducted on an intention-to-treat basis for participants with complete data on relevant measures using SPSS V.18.
Exploratory analyses for parametric data included the paired t-test for within-arm comparisons of mean difference between baseline and 6 months and one-way analysis of varience for between arm comparisons of mean difference. For non-parametric data it included the Wilcoxon signed rank test for within-arm comparisons of median difference and the Kruskal-Wallis test for between arm comparisons of median difference. Categorical data were analysed using the marginal

Table 1 Baseline demographic, socioeconomic and pain data of patients by study arm, prescribing, review and treatment as usual (TAU)

\begin{tabular}{|c|c|c|c|}
\hline & Prescribing* $(n=68)$ & Review* $^{*}(n=62)$ & $\operatorname{TAU}^{*}(n=63)$ \\
\hline Age: mean (SD) & $66.1(12.1)$ & $65.7(14.2)$ & $64.9(11.6)$ \\
\hline Missing & 1 & 1 & 0 \\
\hline Gender (\% female) & $54.4(37)$ & $74.2(46)$ & $58.7(37)$ \\
\hline \multicolumn{4}{|l|}{ Marital status } \\
\hline Married & 43 & 30 & 41 \\
\hline Single & 6 & 6 & 3 \\
\hline Divorced/widow & 10 & 21 & 13 \\
\hline Other & 6 & 4 & 6 \\
\hline Missing & 3 & 1 & 0 \\
\hline \multicolumn{4}{|l|}{ Highest educational level achieved } \\
\hline No qualifications & 30 & 27 & 21 \\
\hline O grade or equivalent & 12 & 6 & 14 \\
\hline Higher/A-level/NVQ3/SVQ3 & 6 & 8 & 7 \\
\hline Tertiary education/NVQ4/NVQ5 & 18 & 17 & 14 \\
\hline Other & 2 & 1 & 4 \\
\hline Missing & 0 & 3 & 3 \\
\hline \multicolumn{4}{|l|}{ Employment status } \\
\hline Employed & 16 & 14 & 9 \\
\hline Unemployed & 3 & 5 & 1 \\
\hline Retired & 38 & 35 & 34 \\
\hline Long-term sick/disabled & 7 & 5 & 9 \\
\hline Other & 3 & 2 & 7 \\
\hline Missing & 1 & 1 & 3 \\
\hline \multicolumn{4}{|l|}{ Household annual income before tax } \\
\hline Less than $£ 9999$ & 13 & 15 & 10 \\
\hline$£ 10000-£ 14999$ & 14 & 18 & 22 \\
\hline$£ 15000-£ 24999$ & 14 & 12 & 12 \\
\hline$£ 25000$-or more & 22 & 11 & 8 \\
\hline Missing & 5 & 6 & 11 \\
\hline \multicolumn{4}{|l|}{ Ethnic group } \\
\hline Caucasian & 67 & 62 & 61 \\
\hline Other & 1 & & \\
\hline Missing & 0 & 0 & 2 \\
\hline \multicolumn{4}{|l|}{ Pain duration (years) } \\
\hline$<1$ & 3 & 2 & 4 \\
\hline $1-3$ & 12 & 12 & 7 \\
\hline $3-5$ & 10 & 13 & 9 \\
\hline $5-10$ & 17 & 13 & 15 \\
\hline$>10$ & 26 & 22 & 28 \\
\hline \multicolumn{4}{|l|}{ Pain localisation $(\%, n)$} \\
\hline Back & $27.9(19)$ & $32.3(20)$ & $20.6(13)$ \\
\hline Neck, shoulders & $7.4(5)$ & $9.7(6)$ & $9.5(6)$ \\
\hline Limbs or hips & $42.6(29)$ & $30.6(19)$ & $50.8(32)$ \\
\hline Other & $8.8(6)$ & $4.8(3)$ & 7.9 (5) \\
\hline Missing & 9 & 14 & 7 \\
\hline
\end{tabular}

*Denominator based on numbers allocated to the specific arms, minus any exclusions due to protocol violations. 
homogeneity test for within-arm comparisons (with null hypotheis that the distribution of CPG grade or HADS group does not change between baseline and 6 month follow-up) and the $\chi^{2}$ test for between-arm comparisons; analyses reported here are based on 6 month follow-up data (other than for participant experiences). Withinarm effect sizes, expressed in terms of a Pearson correlation coefficient $(r)$ have been calculated using the formulas from Rosenthal. ${ }^{28}$ Effect sizes can be directly compared using Cohen's ${ }^{29}$ criteria of $\mathrm{r}=0.1$ (small effect); $r=0.3$ (medium effect) and $r=0.5$ (large effect).

\section{RESULTS}

\section{Response rates and demography}

Six of the seven practices approached participated. GPs excluded 12\% (392/3281) of patients, mostly those with dementia. There was no statistically significant difference between participants and non-participants in terms of age, gender and index of multiple deprivation. Figure 1 shows the flow of participants through the study. Overall, the consent rate was $25 \%(356 / 1397)$ and the recruitment rate was $14 \%(196 / 1397)$.

Eighty-six per cent of participants $(251 / 289)$ returned baseline questionnaires, of whom 232 were randomised (36 participants were randomised to one of the two intervention arms for training purposes and were not included in any further analysis and 19 were not included as the recruitment target had been met). The overall follow-up rate at 3 months was $86 \%(161 / 187)$ and at 6 months $84 \%(152 / 180)$.

As shown in table 1, groups were similar at baseline for demographic and socioeconomic variables and pain data. Most participants were married, Caucasian and female, older (mean (SD) age 65 (12.6) years), had an annual income of $<£ 25000$ and had suffered from pain for at least 5 years. Most $(57 \% ; 103 / 181)$ reported being fully adherent to their medication regimen (MMAS-4, median 4.0 (IQR 3.0-4.0); 15 missing MMAS scores).

In the prescribing arm, $78 \%(53 / 68)$ attended an initial prescribing consultation, 31 had at least one planned follow-up (of which $34 / 37$ were conducted by phone) and 130 recommendations were made for $92 \%$ $(49 / 53)$ of participants seen. Examples are shown in box 1 . The median time taken for the note-based record review was $35 \mathrm{~min}$ (IQR 20.0, 45.0), the consultation was $30 \mathrm{~min}$ (IQR 20.0, 40.0), careplan preparation $10 \mathrm{~min}$ (IQR 10.0, 20.0) and median duration of follow-ups was 10 min (IQR 5.0-15.0).

In the review arm $97 \%(60 / 62)$ of participants' records were reviewed (note there was one postrandomisation exclusion) for whom 197 recommendations were made. Where GP feedback was provided $(n=48)$, they generally agreed with pharmacists' recommendations, which were fully implemented for 20 participants ( 2 by the pharmacist following request by GP), partially for 19 participants and not at all for nine participants. The median time taken for the note-based record review was
Box 1 Examples of pharmacist interventions in the prescribing arm

Changes to pain management. 'use paracetamol regularly', 'take tramadol if needed' 'add piroxicam gel PRN', 'given web links to self help groups'

Compliance aid: 'gave written times that this drug could be taken' Addressing side effects/safety. 'take paracetamol after initial NSAID', 'take senna', 'ordered blood monitoring', 'stop use of two NSAIDS

General health: 'discussed weight loss', 'invited to practice nurse for BP', 'glucose, lipids and lifestyle update'

Cost minimisation: 'change aspirin EC to plain'.

30 min (IQR 24.3, 45.0), and careplan preparation was $10 \mathrm{~min}$ (IQR 5.0, 20.0).

\section{Clinical outcome measures}

Table 2 shows the mean (SD) or median (IQR) of the CPG for each arm at baseline and 6-month follow-up. Table 3 shows the SF-12 scores and table 4 shows the HADS-A and HADS-D results.

In the prescribing arm, there was a statistically significant within arm improvement for the CPG intensity $(p=0.002$, effect size $(r)=0.45)$ and disability $(p=0.003$, effect size $(r)=0.43)$ subscales and between arms on the intensity subscale $(p=0.02)$, but not the disability subscale ( $\mathrm{p}=0.55$; table 2$)$. There was a significant within-arm improvement in overall CPG grade in the prescribing $(\mathrm{p}=0.003)$ and review arm $(\mathrm{p}=0.001)$, but not in the TAU arm. The SF-12 PCS showed a statistically significant within arm improvement in the TAU arm $(\mathrm{p}=0.02$, effect size $(r)=0.35$; table 3 ), but not between trial arms. The SF-12 MCS showed a statistically significant deterioration in the TAU arm $(p=0.002$, effect size $(r)=0.45$; (table 3$)$, as did the HADS-D ( $\mathrm{p}=0.03$, table 4$)$. Analysis was also carried out on the non-categorised HADS scores which showed a statistically significant improvement within the prescribing arm for Depression $(\mathrm{p}=0.022)$ and Anxiety $(p=0.007)$. These were both significant between groups ( $\mathrm{p}=0.022$ and $\mathrm{p}=0.045$, respectively; table 5 ).

\section{Acceptability of the pharmacist prescribing intervention}

All six pharmacists and $56 \%$ of the GPs (23/41) were interviewed. All pharmacists and most GPs were positive about the intervention, although some GPs suggested that the pharmacists' recommendations had been minor and questioned the cost-effectiveness of the service. Patient participants were generally positive about the pharmacist prescribing service although some concerns were identified, as illustrated by the quotes shown in box 2 .

\section{DISCUSSION}

\section{Principal findings}

This exploratory RCT of pharmacist-led management of patients with chronic pain suggests that pharmacist 
Table 2 Mean (SD) CPG intensity, median (IQR) CPG disability and count CPG grade at baseline, 6-month follow-up and difference between the two assessment points for each arm, prescribing, review and treatment as usual (TAU)

\begin{tabular}{|c|c|c|c|c|c|c|c|}
\hline & $\mathbf{N}^{*}$ & $\begin{array}{l}\text { Prescribing } \\
\text { Mean (SD) }\end{array}$ & $\mathbf{N}^{*}$ & $\begin{array}{l}\text { Review } \\
\text { Mean (SD) }\end{array}$ & $\mathbf{N}^{*}$ & $\begin{array}{l}\text { TAU } \\
\text { Mean (SD) }\end{array}$ & $\begin{array}{l}\text { p Value } \\
\text { (between groups } t \text { ) }\end{array}$ \\
\hline Baseline CPG intensity & 47 & $66.1(16.0)$ & 45 & $68.4(17.6)$ & 54 & $65.4(18.0)$ & \\
\hline 6-month follow-up CPG intensity & & $58.1(19.5)$ & & $67.4(21.7)$ & & $65.6(19.6)$ & \\
\hline Difference CPG intensity & & $-8.0(16.3)$ & & $-1.0(16.0)$ & & $0.2(14.9)$ & \\
\hline p (within groups $\ddagger$ ) & & 0.002 & & 0.67 & & 0.93 & 0.02 \\
\hline \multirow{2}{*}{ Effect size $(r)$} & & 0.45 & & 0.07 & & 0.01 & \\
\hline & & Median (IQR) & & Median (IQR) & & Median (IQR) & \\
\hline Baseline CPG disability & 48 & $60.0(30.0 ; 75.8)$ & 46 & $66.7(45.0 ; 80.0)$ & 53 & $56.7(36.7 ; 80.0)$ & \\
\hline 6-month follow-up CPG disability & & $40.0(20.0 ; 60.0)$ & & $53.3(29.2 ; 73.3)$ & & $50.0(25.0 ; 80.0)$ & \\
\hline Difference CPG disability & & $-8.3(-23.3 ; 0.0)$ & & $-3.3(-16.7 ; 10.0)$ & & $-3.3(-21.7 ; 5.0)$ & \\
\hline p (within groups $\ddagger$ ) & & 0.003 & & 0.15 & & 0.05 & 0.55 \\
\hline Effect size ( $r$ ) & & 0.43 & & 0.20 & & 0.26 & \\
\hline Baseline CPG grade & 44 & Count (\%) & 44 & Count (\%) & 48 & Count (\%) & \\
\hline I & & $5(11.4)$ & & $3(6.8)$ & & $5(10.4)$ & \\
\hline II & & $16(36.4)$ & & $9(20.5)$ & & $13(27.1)$ & \\
\hline III & & 7 (15.9) & & $10(22.7)$ & & $13(27.1)$ & \\
\hline VI & & $16(36.4)$ & & $22(50.0)$ & & $17(35.4)$ & \\
\hline \multicolumn{8}{|l|}{ 6-month follow-up CPG grade } \\
\hline I & & $13(29.5)$ & & 8 (18.2) & & $6(12.5)$ & \\
\hline II & & $13(29.5)$ & & $15(34.1)$ & & $17(35.4)$ & \\
\hline III & & 8 (18.2) & & 8 (18.2) & & $11(22.9)$ & \\
\hline IV & & $10(22.7)$ & & $13(29.5)$ & & $14(29.2)$ & \\
\hline \multicolumn{8}{|l|}{ Difference CPG grade } \\
\hline$\leq-1$ & & $21(47.7)$ & & $17(38.6)$ & & $15(31.2)$ & \\
\hline$\overline{0}$ & & $17(38.6)$ & & $25(56.8)$ & & $25(52.1)$ & \\
\hline$\geq 1$ & & $6(13.6)$ & & $2(4.5)$ & & $8(2.1)$ & 0.16 \\
\hline$p$ (within groupst) & & 0.003 & & 0.001 & & 0.17 & \\
\hline
\end{tabular}

$\mathrm{p}$ Values for within-arm and between-arm differences are also reported.

${ }^{*}$ Number of participants in each group who completed the appropriate part of the CPG at both baseline and follow-up.

†From analysis of variance on mean difference, Kruskall-Wallis on median difference or $\chi^{2}$ test on difference in CPG grade as appropriate.

†From paired $t$ test, Wilcoxon signed rank test or marginal homogeneity test as appropriate.

CPG, Chronic Pain Grade. 


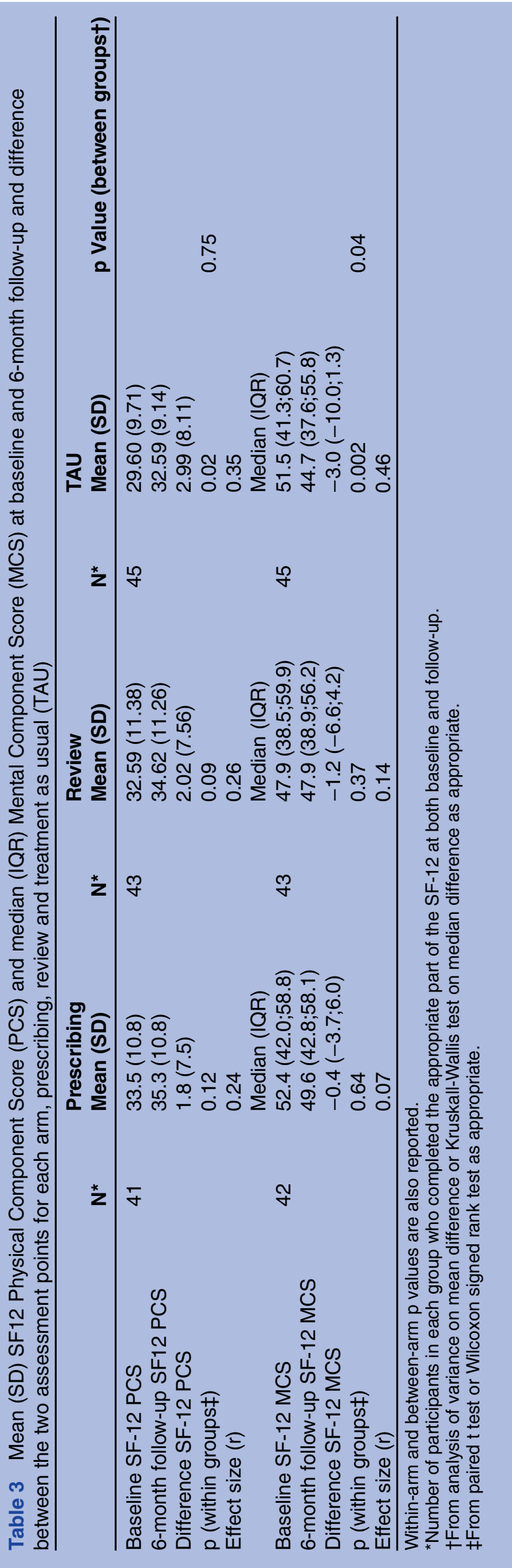

prescribing (and possibly pharmacist review alone) may be effective in improving pain-related outcomes and be acceptable to both patients and most professionals. There was an indication of a positive effect on emotional health, but no measurable effect on general health.

\section{Strengths and weaknesses}

This was the first RCT to assess clinical and humanistic outcomes after pharmacist prescribing for any clinical condition compared with usual GP care, and the first RCT to specifically assess pharmacist-led management of chronic pain, compared with usual GP care. It was based on extensive development and feasibility work ${ }^{14}{ }^{15}$ in line with MRC framework for development and evaluation of complex interventions. ${ }^{30} \mathrm{~A}$ range of validated outcome measures was included, as well as a parallel qualitative process evaluation which assessed satisfaction and acceptability. The inclusion of six practices and their associated pharmacists from both Scotland and England increased the generalisability of the findings. Pharmacists received formal training and agreed and used a common treatment algorithm which should have increased standardisation of treatment. The preponderance of women (overall 62\%) and average age of 65 years reflects the wider chronic pain population ${ }^{1}$ as does the distribution of pain site. ${ }^{31} 32$

There were, however limitations. Although high follow-up response rates were achieved at both $3(86 \%)$ and 6 months $(85 \%)$ only $25 \%$ of eligible patients entered the trial. This low initial consent rate is in line with other studies, ${ }^{33}{ }^{34}$ but may cause unknown biases including problems of generalisability, as does the solely Caucasian ethnicity. Concerns identified by participants during the formal feedback, for example, having too many people involved in one's care may have contributed to poor response rates and rewording of participant recruitment documentation to reassure participants of the role of the pharmacist could address this. More participants withdrew in the prescribing arm compared with the other two arms, which might be attributed to the need for an additional practice visit. The study was an exploratory trial so no formal power calculation was undertaken. However, because there were no published MIDs available to estimate effect size for the outcomes in this population, it was important to present the actual clinical magnitude of change in outcome at 6 months alongside a statistical assessment of this change ( $p$ value). This allows an assessment of both clinical and statistical significance simultaneously with the caveat that this is an exploratory study. With around 50 patients per arm, this was deemed sufficient numbers to examine the change in outcome measures with appropriate within-group and between-group univariate statistical tests. Owing to the nature of the intervention, no participants were blind to their group allocation, and so some outcomes, especially the qualitative components, may have been affected by social desirability bias. 
Table 4 The Hospital Anxiety and Depression Scale-Depression (HADS-D) and HADS-Anxiety (HADS-A) count of patients according to severity (normal, mild, moderate or severe) and the difference in severity category between the two assessment points for each arm, prescribing, review and treatment as usual (TAU)*

\begin{tabular}{|c|c|c|c|c|c|c|c|}
\hline & $\mathbf{N}^{*}$ & Prescribing & $\mathbf{N}^{*}$ & Review & $\mathbf{N}^{*}$ & TAU & $\begin{array}{l}\text { p Value } \\
\text { (between groups } †\end{array}$ \\
\hline Baseline HADS-D & 44 & Count (\%) & 45 & Count (\%) & 53 & Count $(\%)$ & \\
\hline Normal & & $32(72.7)$ & & $31(68.9)$ & & $38(71.7)$ & \\
\hline Mild & & $8(18.2)$ & & $11(24.4)$ & & $7(13.2)$ & \\
\hline Moderate & & $3(6.8)$ & & $3(6.7)$ & & $8(15.1)$ & \\
\hline Severe & & $1(2.3)$ & & 0 & & 0 & \\
\hline \multicolumn{8}{|c|}{ 6-month follow-up HADS-D } \\
\hline Normal & & $32(72.7)$ & & $32(71.1)$ & & $32(60.4)$ & \\
\hline Mild & & 7 (15.9) & & $6(13.3)$ & & $10(18.9)$ & \\
\hline Moderate & & 5 (11.4) & & $6(13.3)$ & & $8(15.1)$ & \\
\hline Severe & & 0 & & $1(2.2)$ & & $3(5.7)$ & \\
\hline \multicolumn{8}{|l|}{ Difference HADS-D } \\
\hline$\leq-1$ & & $5(11.4)$ & & $4(8.9)$ & & $2(3.8)$ & \\
\hline$\overline{0}$ & & 34 (77.3) & & $37(82.0)$ & & $40(75.5)$ & \\
\hline$\geq 1$ & & 5 (11.4) & & $4(8.9)$ & & $11(20.8)$ & 0.32 \\
\hline$p$ (within groups $\ddagger$ ) & & 1.0 & & 0.71 & & 0.03 & \\
\hline Baseline HADS-A & 44 & Count (\%) & 43 & Count (\%) & 48 & Count (\%) & \\
\hline Normal & & $25(56.8)$ & & $30(69.8)$ & & $29(60.4)$ & \\
\hline Mild & & 8 (18.2) & & $7(16.3)$ & & $9(18.8)$ & \\
\hline Moderate & & 8 (18.2) & & $5(11.6)$ & & $8(16.7)$ & \\
\hline Severe & & $3(6.8)$ & & $1(2.3)$ & & $2(4.2)$ & \\
\hline \multicolumn{8}{|c|}{ 6-month follow-up HADS-A } \\
\hline Normal & & $27(61.4)$ & & $29(67.4)$ & & $32(66.7)$ & \\
\hline Mild & & 7 (15.9) & & $6(14.0)$ & & $5(10.4)$ & \\
\hline Moderate & & 8 (18.2) & & $6(14.0)$ & & $10(20.8)$ & \\
\hline Severe & & $2(4.5)$ & & $2(4.7)$ & & $1(2.1)$ & \\
\hline \multicolumn{8}{|l|}{ Difference HADS-A } \\
\hline$\leq-1$ & & $6(13.6)$ & & $3(7.0)$ & & $10(20.8)$ & \\
\hline$\overline{0}$ & & $35(79.5)$ & & $33(76.7)$ & & $29(60.4)$ & \\
\hline$\geq 1$ & & $3(6.8)$ & & 7 (16.3) & & $9(18.8)$ & 0.14 \\
\hline $\bar{p}$ (within groups $\ddagger$ ) & & 0.25 & & 0.21 & & 0.55 & \\
\hline
\end{tabular}

Within-arm and between-arm $p$ values are also reported.

*Number of participants in each group who completed the appropriate part of the HADS at both baseline and follow-up.

†From $\chi^{2}$ test on difference in HADS.

†From marginal homogeneity test.

Table 5 Median Hospital Anxiety and Depression Scale-Depression (HADS-D) and HADS-Anxiety (HADS-A) scores (IQR) at baseline and 6-month follow-up and difference between the two assessment points for each arm, prescribing, review and treatment as usual (TAU)

\begin{tabular}{|c|c|c|c|c|c|c|c|}
\hline & & Prescribing & & Review & & TAU & \\
\hline & $\mathrm{N}^{*}$ & Median (IQR) & $\mathrm{N}^{*}$ & Median (IQR) & $N^{*}$ & Median (IQR) & $\begin{array}{l}\mathrm{p} \text { Value } \\
\text { (between groups) }\end{array}$ \\
\hline Baseline HADS-D & 42 & $5.0(3.0 ; 8.0)$ & 44 & $4.5(2.3 ; 8.0)$ & 51 & $5.0(3.0 ; 8.0)$ & \\
\hline 6-month follow-up HADS-D & & $4.0(2.0 ; 8.0)$ & & $5.0(2.0 ; 8.8)$ & & $5.0(2.0 ; 10.0)$ & \\
\hline Difference HADS-D & & $-1.0(-2.0 ; 0.0)$ & & $0.0(-1.0 ; 1.8)$ & & $0.0(-1.0 ; 2.0)$ & 0.02 \\
\hline p (within groups) & & 0.02 & & 0.33 & & 0.22 & \\
\hline Baseline HADS-A & 44 & $7.0(3.3 ; 10.8)$ & 43 & $5.0(3.0 ; 10.0)$ & 48 & $6.0(4.0 ; 10.0)$ & \\
\hline 6-month follow-up HADS-A & & $5.0(2.3 ; 9.8)$ & & $6.0(3.0 ; 9.0)$ & & $7.0(4.0 ; 10.0)$ & \\
\hline Difference HADS-A & & $-1.0(-2.0 ; 0.0)$ & & $0.0(-2.0 ; 2.0)$ & & $0.5(-3.0 ; 2.0)$ & 0.05 \\
\hline$p$ (within groups) & & 0.01 & & 0.45 & & 0.81 & \\
\hline
\end{tabular}

Within-arm and between-arm $p$ values are also reported.

${ }^{*}$ Number of participants in each group who completed the appropriate part of the HADS at both baseline and follow-up. 
Box 2 Examples of quotes from pharmacists ( $n=6)$, general practitioners (GPS) $(n=23)$ and patient participants $(n=40)$ on the prescribing intervention

\section{Pharmacists (from interviews):}

Satisfying $(n=6):$ :contact with patients', 'being able to help patients', 'being able to make a difference to long-standing pain'...'even in small ways'

Interesting $(\mathrm{n}=6)$ :'Iearning about pain'

Challenging $(\mathrm{n}=6)$ :'complex, chronically ill patients'

GPs (from interviews):

Support for the service $(n=17)$ : it's been a very positive thing

Agreement with pharmacists' recommendations $(\mathrm{n}=23)$ : 'oh

very reasonable suggestions', 'tinkering round the edges', 'had been tried already'.

Trust in the practice pharmacist $(\mathrm{n}=23): 4$ respect his professional judgement'

Cost effectiveness $(n=6)$ : 'if there's limited resources do we want to spend the money on a pharmacist'.

Patients (from 3-month questionnaire):

Closed questions:

Proportion agreeing that:

The pharmacist was interested in them $(89 \% ; 39 / 44)$

They were totally satisfied $(85 \% ; 39 / 46)$

They were told about their treatment $(82 \% ; 38 / 46)$

Their consultation was thorough $(79 \% ; 34 / 44)$

They would have liked more time $(9 \% ; 4 / 44)$

They would have preferred to see their GP $(9 \% ; 4 / 44)$

Too many people were now involved in their treatment

(11\%; 5/44).

Open text questions:

Positive $(n=39)$ : 'She was professional, relaxed, pleasant and interested. Excellent!'

Negative $(n=1)$ : 'A waste of time, altered my tablets which made my pain worse'.

Our outcome measures were self-reported, but this is the norm in pain studies as pain is a subjective experience. ${ }^{18}$ Furthermore we do not know how important the observed differences were to participants. Following precedents set in previous research, ${ }^{25}$ and because there is no consensus on an alternative measure ${ }^{35}$ we used the HADS as a tool to classify people by severity of depression and anxiety. However it is strictly a screening tool, and the four levels of severity have not been formally validated. We therefore also compared outcomes using it as a continuous scale.

\section{Relationship with other studies}

This study is important because no other RCT has evaluated pharmacist prescribing and few studies, and importantly no RCTs, have evaluated pharmacist interventions for pain. In pharmacist prescribing most research has focused on reported experiences of professionals and patients, and not used validated outcome measures. Yet pharmacist prescribing is now widely practised. For pain, there have been a few small studies. Briggs et $a l^{36}$ conducted a small before-and-after evaluation (involving 65 patients) of a nurse and pharmacist-led chronic pain clinic in primary care. Pain intensity visual analogue scale scores reduced significantly over 6 months. Another evaluation of 26 patients using a medication review service provided jointly by a physiotherapist and pharmacist in the UK, reported improvement in pain control for $88 \%$ of patients. ${ }^{37}$

The CPG was found to show a graded effect across the three arms, showing discrimination with both direction and strength of improvement, suggesting maximum benefit for those in the pharmacist prescribing arm. However, the reduction in overall score appears to be mediated by a change in the intensity of pain subscale rather than in pain-related disability. The effect size of 0.45 suggests this could be an important difference. In contrast, the SF-12, a measure of general health and functionality showed no significant difference between intervention arms, reflecting either no effect or or lack of power to detect an effect.

While most participants in this study were already within the normal range on the HADS scale, and therefore had minimal chance of improvement, there were nonetheless suggestions of better ourcomes in participants in the prescribing arm. Including a range of instruments is in line with IMMPACT recommendations, ${ }^{38}$ which state that focus should be on the whole person, not just about pain. However, this needs to be balanced with minimising participant burden.

\section{Explanations, implications and future research}

The number of pharmacist's recommendations per participant was higher in the review arm than in the prescribing arm. This might seem contradictory to the possible greater benefit found in the prescribing arm. However, in the prescribing arm pharmacists met the participant and may have more readily identified and dismissed suggestions previously tried. The interview feedback highlighted that some recommendations for change, while sensible, had been tried already. This might also be the reason why there were only $60 \%$ of pharmacist recommendations with which the GP fully agreed. Self-reported adherence to medication at baseline was good. Despite this, the pharmacists still improved pain outcomes in the prescribing arm. This could have been due to changes in medications and/or participant education about optimal timing for administration of analgesic medicines. Further research is needed to confirm the beneficial effect of pharmacist prescribing and its sustainability.

\section{CONCLUSION}

Our results suggest that pharmacist prescribing (and possibly pharmacist review alone) for patients with chronic pain is feasible, acceptable and may lead to improvements in pain and other measures. A larger fully powered trial is now needed to confirm these findings. 
Author affiliations

${ }^{1}$ Health Services Research Unit, Centre for Healthcare Randomised Trials, University of Aberdeen, Aberdeen, UK

${ }^{2}$ Division of Applied Health Sciences, Centre of Academic Primary Care, University of Aberdeen, Aberdeen, UK

${ }^{3}$ Medical Statistics Team, Division of Applied Health Sciences, University of Aberdeen, Aberdeen, UK

${ }^{4}$ Health Economics Research Unit, University of Aberdeen, Aberdeen, UK ${ }^{5}$ Division of Population Health Sciences, University of Dundee, Ninewells Hospital and Medical School, Dundee, UK

${ }^{6}$ Faculty of Medicine and Health Sciences, Norwich Medical School, University of East Anglia, Norwich, UK

${ }^{7}$ School of Pharmacy, University of East Anglia, Norwich, UK

Acknowledgements We thank the participating patients, practices and pharmacists. We would also like to thank Annie Blyth who was the lead researcher in East Anglia, Kirsten Harrild (Medical Statistics, UOA) for statistical support. Rick Adams (School of Pharmacy, UEA) helped design and deliver the pharmacist training and Lesley Thomson (NHS Grampian) helped design the pharmacist data collection forms. The patient postal questionnaire was based on work by Nicola Cooper and the Norfolk Arthritis Register (NOAR) research team. The Pharmacy-Led Management of Chronic Pain Study Team acknowledges the financial support of NHS Research Scotland (NRS), through Scottish Primary Care Research Network Northeast. The work was conducted as part of the Aberdeen Pain Research Collaboration. All authors had access to all of the study data and can take responsibility for the integrity of the data and the accuracy of the data analysis.

Contributors CMB was the chief investigator, conceived the study and led the writing of proposal for funding, provided day to supervision of study conduct, analysis and interpretation of data and co-led the writing of the paper, including revisions and final draft. AME, PCH, RH, AJL, PM, BHS, DW and MW were coinvestigators, contributed to writing of the proposal, oversight of the study conduct, data analysis and interpretation, drafting of the paper and approved the final version. PM had particular responsibility for the health economic components and AJL for the statistical analyses. BHS and AME provided specific advice on chronic pain and it measurement and DW expertise on the design and delivery of the pharmacist training. HB was the research fellow, coordinated the two study sites and was lead researcher for Grampian. She was responsible for daily study conduct and coordination, acquisition of the data, analysis, producing tables and figures and interpretation of data. She co-led the drafting of the paper with CMB and approved the final version.

Funding The project was funded by the Medical Research Council (grant ID: 85356). They had no further involvement in any aspect of study conduct; all researchers were independent of the funding body.

\section{Competing interests None.}

Ethics approval North of Scotland.

Provenance and peer review Not commissioned; externally peer reviewed.

Data sharing statement Consent was not obtained from participants for data sharing and so cannot be released to third parties. We welcome, however, opportunities to work collaboratively with other investigators to use the data for further analyses.

\section{REFERENCES}

1. Elliott AM, Smith BH, Penny KI, et al. The epideomiology of chronic pain in the community. Lancet 1999;354:1248-52.

2. Elliott AM, Smith BH, Hannaford $P$, et al. The course of chronic pain in the community: results of a 4-year follow-up study. Pain 2002:99:299-307.

3. Becker N, Bondegaard Thomsen A, Olsen AK, et al. Pain epidemiology and health related quality of life in chronic non-malignant painpain center patients referred to a Danish multidisciplinary. Pain 1997;73:393-400.

4. Breivik H, Collett B, Ventafridda V, et al. Survey of chronic pain in Europe: prevalence, impact on daily life, and treatment. Eur $\mathrm{J}$ Pain 2006;10:287-333.
5. Gureje O, Von Korff M, et al., Persistent pain and well-being: a World Health Organization Study in Primary Care. JAMA 1998;280:147-51.

6. Magni G, Marchetti M, Moreschi C, et al. Chronic musculoskeletal pain and depressive symptoms in the National Health and Nutrition Examination. I. Epidemiologic follow-up study. Pain 1993;53:163-8.

7. Sullivan MD, Turner JA, Romano J. Chronic pain in primary care: identification and management of psychological factors. J Fam Pract 1991;32:193-9.

8. Green S, Buchbinder R, et al. Physiotherapy interventions for shoulder pain. Cochrane Database Syst Rev 2010(9): CD004258.

9. Eccleston C, Williams ACDC, Morley S. Psychological therapies for the management of chronic pain (excluding headache) in adults. Cochrane Database Syst Rev 2009;(2):CD007407.

10. Haetzman M, Elliott AM, Smith BH, et al. Chronic pain and the use of conventional and alternative therapy. Fam Pract 2003;20:147-54.

11. Pirmohamed $M$, James $S$, Meakin $S$, et al. Adverse drug reactions as cause of admission to hospital: prospective analysis of 18820 patients. BMJ 2004;329:15.

12. Krska J, Cromarty JA, Arris F, et al. Pharmacist-led medication review in patients over 65: a randomized, controlled trial in primary care. Age Ageing 2001;30:205-11.

13. Department of Health. Improving patients' access to medicines: a guide to implementing nurse and pharmacist independent prescribing within the NHS England. Department of Health, 2006 http://www.prescribingforsuccess.co.uk/document uploads/About/ DHGuideApril06.pdf

14. McDermott ME, Smith BH, Elliott AM, et al. The use of medication for chronic pain in primary care, and the potential for intervention by practice-based pharmacist. Fam Pract 2006;23:46-52.

15. Bruhn $\mathrm{H}$, Bond CM, Elliott AM, et al. Developing an RCT of general practice-based, pharmacist-led, management of chronic pain: the PIPPC study. IJPP 2010;18(Suppl 2):23.

16. Smith BH, Read JRM, Chambers WAC, et al. Researching chronic pain: identification of a community based sample. Pain Clin 1996;9:73-6.

17. Department of Health. Supplementary prescribing by nurses and pharmacists within the NHS in England: a guide for implementation. Department of Health, 2003. http://webarchive.nationalarchives.gov. uk/+/www.dh.gov.uk/en/Publicationsandstatistics/Publications/ PublicationsPolicyAndGuidance/DH_4009717

18. Turk DC, Dworkin RH, Allen RR, et al. Core outcome domains for chronic pain clinical trials: IMMPACT recommendations. Pain 2003;106:337-45.

19. Ware JE, Kosinski M, Turner-Bowker DM, et al. User's manual for the SF-12v2 health survey. 2nd edn. QualityMetric, Incorporated, 2009.

20. Nicholl BI, Macfarlane GJ, Davies KA, et al. Premorbid psychosocial factors are associated with poor health-related quality of life in subjects with new onset of chronic widespread pain — results from the EPIFUND study. Pain 2009;141:119-26.

21. Carmona L, Ballina J, Gabriel R, et al. The burden of musculoskeletal diseases in the general population of Spain: results from a national survey. Ann Rheum Dis 2001;60:1040-5.

22. Feeny D. Preference-based measures: utility and quality-adjusted life years. In: Fayers P, Hays R., eds. Assessing quality of life in clinical trials. 2nd edn. Oxford: Oxford University Press, 2005:405-29, Chapter 6.2.

23. Von Korff FJ, Ormel M, Keefe J, et al., Grading the severity of chronic pain. Pain 1992;50:133-49.

24. Zigmond AS, Snaith RP. The hospital anxiety and depression scale. Acta Psychiatr Scand 1983;67:361-70.

25. Snaith RP, Zigmond AS. HADS: Hospital anxiety and depression scale. Windsor: NFER Nelson, 1994.

26. Morisky DE, Green LW, Levine DM. Concurrent and predictive validity of a self-reported measure of medication adherence. Med Care 1986;24:67-74.

27. Lancaster GA, Dood S, Williamson PR. Design and analysis of pilot studies: recommendations for good practice. J Eval Clin Pract 2004; $10: 307-12$.

28. Rosenthal R. Meta-analytic procedures for social research (revised). Newbury Park, CA: Sage, 1991.

29. Cohen J. Statistical power analysis for the behavioural sciences. 2nd edn. New York: Academic Press, 1988.

30. Craig P, Dieppe P, Macintyre S, et al. Developing and evaluating complex interventions: the new Medical Research Council guidance. BMJ 2008;337:a1655

31. Magni M, Caldieron C, Rigatti-Luchini S, et al. Chronic musculo-skeletal pain and depressive symptoms in the genera 
population. An analysis of the 1st National Health and Nutrition Examination Survey data. Pain 1990;43:299-307.

32. Gureje O, Von Korff M, et al., Persistent pain and well-being A World Health Organization study in primary care. JAMA 1998;280:147-51.

33. Community Pharmacy Medicines Management Project Evaluation Team. The MEDMAN study: a randomized controlled trial of community pharmacy-led medicines management for patients with coronary heart disease. Fam Pract 2007;24:189-200.

34. Treweek S, Mitchell E, Pitkethly M, et al. Strategies to improve recruitment to randomised controlled trials. Cochrane Database Syst Rev 2010;(4):MR0000132011.
35. Cameron IM, Cardy A, Crawford JR, et al. Measuring depression severity in general practice: discriminatory performance of the PHQ-9, HADS-D, and BDI-II. Br J Gen Pract 2011;61:e419-26.

36. Briggs $\mathrm{M}$, Closs SJ, Marczewski $\mathrm{K}$, et al. A feasibility study of a combined nurse/pharmacist-led chronic pain clinic in primary care. Qual Prim Care 2008;16:91-4.

37. Anonymous. Pharmacist and physiotherapist-led community outreach pain programme improve quality of life. Pharm $J$ 2005;275:14.

38. Turk DC, Dworkin RH, Revicki D, et al. Identifying important outcome domains for chronic pain clinical trials: an IMMPACT survey of people with pain. Pain 2008;137:276-85. 